\title{
Scalar transport from a point source in flows over wavy walls
}

\author{
Carsten Wagner · Simon Kuhn • \\ Philipp Rudolf von Rohr
}

Received: 29 September 2006/Revised: 10 May 2007/ Accepted: 31 May 2007 / Published online: 11 July 2007

(C) Springer-Verlag 2007

\begin{abstract}
Simultaneous measurements of the velocity and concentration field in fully developed turbulent flows over a wavy wall are described. The concentration field originates from a low-momentum plume of a passive tracer. PLIF and digital particle image velocimetry are used to make spatially resolved measurements of the structure of the scalar distribution and the velocity. The measurements are performed at three different Reynolds numbers of $R e_{\mathrm{b}}=$ $5,600, R e_{\mathrm{b}}=11,200$ and $R e_{\mathrm{b}}=22,400$, respectively, based on the bulk velocity $u_{\mathrm{b}}$ and the total channel height $2 h$. The velocity field and the scalar field are investigated in a water channel with an aspect ratio of 12:1, where the bottom wall of the test section consists of a train of sinusoidal waves. The wavy wall is characterized by the amplitude to wavelength ratio $\alpha=0.05$ and the ratio $\beta$ between the wave amplitude and the half channel height where $\beta=0.1$. The scalar is released from a point source at the wave crest. For the concentration measurements, Rhodamine B is used as tracer dye. At low to moderate Reynolds number, the flow field is characterized through a recirculation zone which develops after the wave crest. The recirculation zone induces high intensities of the fluctuations of the streamwise velocity and wall-normal velocity. Furthermore, large-scale structures are apparent in the flow field. In previous investigations it has been shown that these largescale structures meander laterally in flows over wavy bottom walls. The investigations show a strong effect of the wavy bottom wall on the scalar mixing. In the vicinity of the source, the scalar is transported by packets of fluid with a high scalar concentration. As they move downstream,
\end{abstract}

C. Wagner $\cdot$ S. Kuhn $\cdot$ P. Rudolf von Rohr $(\bowtie)$

Institute of Process Engineering, ETH Zurich,

8092 Zurich, Switzerland

e-mail:philippr@ethz.ch these packets disintegrate into filament-like structures which are subject to strong gradients between the filaments and the surrounding fluid. The lateral scale of the turbulent plume is smaller than the lateral scale of the large-scale structures in the flow field and the plume dispersion is dominated by the structures in the flow field. Due to the lateral meandering of the large-scale structures of the flow field, also the scalar plume meanders laterally. Compared to turbulent plumes in plane channel flows, the wavy bottom wall enhances the mixing effect of the turbulent flow and the spreading rate of the scalar plume is increased.

\section{Introduction}

Most engineering and geophysical flows show some common features like flow separation, streamline curvature, and interaction of the flow with a (complex) surface geometry. They are more complex than simple building block flows. Furthermore, engineering and geophysical flows are often associated with an additional transport of a passive or active scalar. The flow over a wavy wall can resemble some of these essential features like flow separation, streamline curvature, and complex interactions between the flow and a surrounding surface.

The present study investigates the influence of a wavy boundary on scalar transport from a point source located at the wave crest. The bottom geometry is defined as

$y_{\mathrm{w}}(x)=a \sin \left(\frac{2 \pi x}{\Lambda_{\mathrm{w}}}\right)$,

where $a$ denotes the wave amplitude, and $\Lambda_{\mathrm{W}}$ stands for the wave length. The geometry of a channel with a wavy 
bottom wall then can be characterized by two dimensionless groups which are the ratio $\alpha$ between the wave amplitude $a$ and the wave length $\Lambda_{\mathrm{w}}$

$\alpha=\frac{a}{\Lambda_{\mathrm{w}}}$

and the ratio $\beta$ between the wave amplitude and the channel half height $h$ :

$\beta=\frac{a}{h}$

The actual values in the present experiments are $\alpha=0.05$ and $\beta=0.1$, respectively. The flow field itself can be characterized by the Reynolds number

$R e_{\mathrm{b}}=\frac{2 h u_{\mathrm{b}}}{v}$

with the bulk velocity

$u_{\mathrm{b}}=\frac{1}{2 h \Lambda_{\mathrm{w}}} \int_{0}^{\Lambda_{\mathrm{w}}} \int_{y_{\mathrm{w}}}^{2 h} u(x, y) \mathrm{d} y \mathrm{~d} x$,

the total channel height $2 h$, and the kinematic viscosity $v$.

Since the near-wall regions of boundary layers and channel flows are quite similar (Sreenivasan 1989)_whereas the outer layers and some of the instantaneous features are not - there might be some universality in the statistical results from wall bounded flows which are spanwise homogeneous (George 2007). If the channel half height $h$ is replaced by the boundary layer thickness $\delta$ in Eq. 3 the present geometry lies in a range of the parameters $\alpha$ and $\beta$ which is common in flows over low hills (Boyer and Davies 2000). Gong and Ibbetson (1989) and Gong (1991) used $\beta \approx 0.1$ in their wind tunnel study of flow over hills. The flow over a wavy bottom surface-the top surface either rigid or a free surface-has been used as a model for geophysical flows. Krettenauer and Schumann (1992) and Maaß and Schumann (1996) used the flow over a wavy wall with similar ratios $\alpha$ and $\beta$ as in the present study to investigate atmospheric flows (the convective boundary layer with and without mean wind) over hilly terrain. In the studies of Calhoun and Street (2001) and Chang and Scotti (2003) the flow over wavy wall is used as a model for currents over ripples in oceanography.

Previous investigations on flows over wavy boundaries assessed the flow field as well as the scalar field. In flows over a wavy wall intermittent and time-mean flow reversal can occur for $\alpha>0.016$ and appropriate Reynolds numbers which was extensively studied by Zilker and Hanratty (1979). The separated flow over wavy walls can be decomposed into four zones which are an outer flow, a shear layer, a separated region and a thin boundary layer (Hudson et al. 1996). The boundary layer is formed after the reattachment of the flow. Günther and Rudolf von Rohr (2003) showed the existence of large-scale structures in flows over a wavy boundary with $\alpha=0.05$ and $\beta=0.1$. The large-scale structures showed a characteristic spanwise scale of $\mathcal{L}_{\mathrm{z}} \approx 3 h$. These large-scale structures meander laterally (Kruse et al. 2003) and they are assumed to carry the bulk of the momentum transport in flows over such a wavy wall, see also Hussain (1983).

Wavy walls are able to alter the scalar transport compared to a plane channel or a flat plate boundary layer. The altered flow structures due to complex surfaces are able to enhance the turbulent transport of scalars from distributed sources and therefore to increase the stirring and mixing processes (Günther and Rudolf von Rohr 2002; Kruse and Rudolf von Rohr 2006).

Scalar statistics for ground level point sources in turbulent boundary layers were investigated by Fackrell and Robins (1982). It was shown that the maximum of production of concentration fluctuations occurs near the source. For ground-level sources, the maximum of the mean concentration $\langle c\rangle / c_{0}$ is always at the surface for all streamwise distances. Similar results were found by Crimaldi and Koseff (2001) and Crimaldi et al. (2002). The dispersion of a vertically constrained plume was investigated by Fong and Stacey (2003). They observed lateral meandering of a low-momentum ground level scalar plume. From their results it can be concluded that large-scale structures in the flow field determine the lateral variability of the plume centreline. Classic theory assumes that the scalar plume is affected by flow structures of different scales. Meandering is caused by velocity fluctuations with scales larger than the plume whereas the dispersion is dominated by velocity scales comparable to the plume size.

\section{Experimental facilities and measurement techniques}

\subsection{Channel flow facility}

The experiments are conducted in a closed-loop flow channel facility designed for investigations of turbulent flows at a wide range of Reynolds numbers using light sheet techniques. A detailed description of the flow channel facility can be found in Günther (2001). A schematic of the facility is shown in Fig. 1. The volume of the working fluid is approximately $0.280 \mathrm{~m}^{3}$ and consists of filtered and de-ionized water. A frequency controlled centrifugal pump (10) with an electric power of $P=6.5 \mathrm{~kW}$ is used in the current experiments. In the loop, apparent pulsations of the flow are sufficiently 


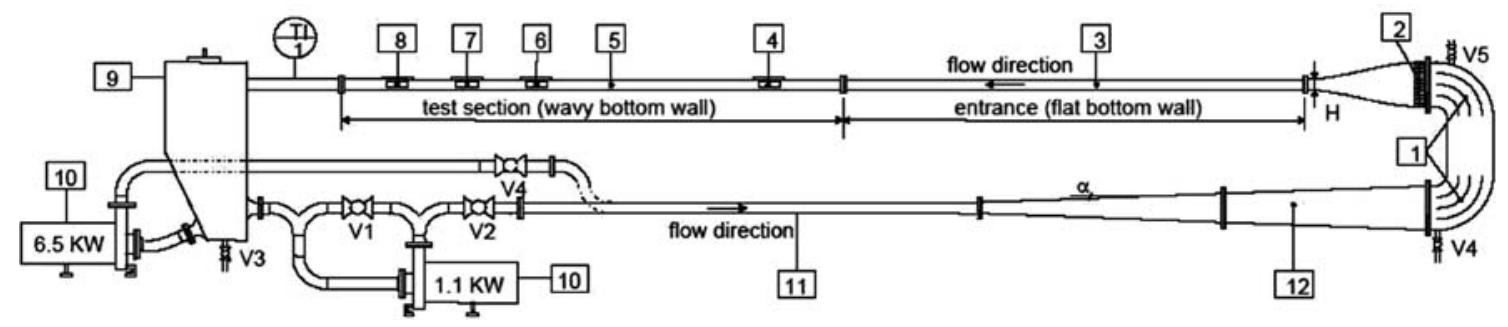

Fig. 1 Closed-loop flow channel facility used in the experiments. The measurements were taken at location (7)

minimized through the plenum chamber and the settling chamber (9). With the current pump a maximum mean bulk velocity of $u_{\mathrm{b}}=1.3 \mathrm{~m} / \mathrm{s}$ is obtainable. The tubing at the pressure side of the pump (with a circular cross section and an inner diameter of $d_{\mathrm{i}}=50 \mathrm{~mm}$ ) is connected to a 2,250 $\mathrm{mm}$ long diffuser with a maximum opening angle of $1.9^{\circ}$. Turning vanes (1) change the flow direction. After passing a hexagonal honeycomb (2) with a lengthto-cell-size ratio of 7 a flow with low turbulence intensity and a uniform velocity distribution is obtained by using a contractor with a contraction-area-ratio of 6.7 immediately after the turning vanes. A boundary layer trip is located at the beginning of the entrance section. In the entrance section and the test section the total channel height is $2 h=30 \mathrm{~mm}$ and the width is $W=360 \mathrm{~mm}$. The aspect ratio of the two sections is $W / 2 h=12: 1$ which ensures measurements undisturbed by the side walls in the center of the test section (e.g., Brederode and Bradshaw 1978). The length of the entrance section and the test section is 2,010 and $2,160 \mathrm{~mm}$, respectively. In the flow loop, the test section is removable to allow for investigations with different bottom geometries.

The bottom wall of the test section itself consists of a train of 72 sinusoidal waves with a wavelength $\Lambda_{\mathrm{w}}=$ $30 \mathrm{~mm}$ (wave amplitude $a=1.5 \mathrm{~mm}$ ) whereas the top wall is flat. The wave length equals the total channel height $2 h$. The measurements are carried out at Reynolds numbers of 5,600, 11,200 and 22,400. Measurements are performed at location (7), see Fig. 1 after 50 wave lengths over which the flow establishes a fully developed turbulent state. The flush mounted point source is located at the wave crest and is designed as a low momentum source. The source consisted of a stainless steel tube (integrated in the bottom wall) with an inner diameter of $d_{\mathrm{i}}=0.8 \mathrm{~mm}$ connected to a calibrated syringe pump.

\subsection{Simultaneous measurements}

We use two techniques to establish the instantaneous and statistical properties of the flow field, the scalar field and the turbulent scalar fluxes. The simultaneous measurements of the velocity field and the concentration field are performed by means of a combined digital particle image velocimetry (DPIV) (Adrian 1991; Westerweel 1997) and planar laser-induced fluorescence (PLIF) technique (e.g., Walker 1987). The measurements are carried out in two different orthogonal planes, the $(x, y)$-plane (center plane of the channel) and the $(x, z)$-plane. The light sheet for the $(x, z)$-plane is located shortly above the wave crest at a distance of $2 \mathrm{~mm}$ from the top of the hill.

\subsubsection{Velocity measurements}

The velocity field in the $(x, y)$-plane is assessed by means of particle image velocimetry (PIV) whereas stereoscopic particle image velocimetry (stereo-PIV) is used in the $(x, z)$-plane.

PIV in the $(x, y)$-plane The PIV measurements in the $(x, y)$-plane are performed by means of a PCO SensicamQE with a pixel resolution of $1,376 \times 1,040$ pixel $^{2}$ in combination with a Nikon Micro Nikkor $60 \mathrm{~mm}, f / 2.8$ lens. The PCO SensicamQE allows a frame rate of $5 \mathrm{~Hz}$ (double images per second). The field-of-view is illuminated by a flash lamp pumped dual cavity Nd:YAG laser (Solo III PIV, New Wave Research) with a maximum output power of $50 \mathrm{~mJ} /$ pulse and a maximum repetition rate of $15 \mathrm{~Hz}$. The laser sheet is generated by means of a cylindrical lens and two spherical lenses. The lenses shape the laser beam to a sheet with a thickness of approximately $t_{1} \approx 0.25 \mathrm{~mm}$ which is a compromise between the needs of the PIV and PLIF.

The area-of-view is $42 \times 32 \mathrm{~mm}^{2}$ in the streamwise and vertical direction, respectively. One pixel represents therefore a square with an edge length of approximately $30.5 \mu \mathrm{m}$ in the measurement area. Seeding is achieved by use of polyamide spheres with a mean diameter $d_{\mathrm{m}}=$ $50 \mu \mathrm{m}$ (PSP-50, Dantec Dynamics) and the seeding density is adjusted to a count of three to five particles per interrogation area (Keane and Adrian 1992; Westerweel 2000). The time between two laser pulses is optimized for a particle shift of 6-8 pixels (Raffel et al. 2000).

Raw images for PIV have been processed by using an adaptive cross-correlation technique. The algorithm used is 
based on window shifting and deformation (Scarano 2002). A first displacement field is obtained by means of a conventional cross-correlation with an interrogation area size of $32 \times 32$ pixel $^{2}$. The results from the cross correlation where filtered by means of a signal-to-noise filter and a window velocity filter which resulted in less than $5 \%$ spurious vectors. The following steps use the window deformation and shifting technique. In difference to the algorithm proposed by Scarano (2002), B-splines are used for sub-pixel shifting. The final correlation results in an interrogation area size of $16 \times 16$ pixel $^{2}$ covering an area of approximately $0.488 \times 0.488 \mathrm{~mm}^{2}$ in the object plane. An overlap factor of $50 \%$ is applied between adjacent windows. At the end, the amount of invalid data is below $2 \%$.

Typical uncertainty sources in PIV data are the statistical uncertainty, resolution uncertainty (subgrid pixel accuracy), calibration uncertainty, and data filtering uncertainty. The first two sources of uncertainty are random errors and the uncertainty can be reduced by using a large number of image pairs. Statistical analysis has shown that a number of 1,000 image pairs is sufficient to satisfy a $95 \%$-confidence criteria. The general uncertainty of the velocity measurements can be estimated to be less than $1 \%$ in the present study resulting from the used algorithm and the experimental setup. The uncertainty of the Reynolds shear stress measurements is somewhat higher and is approximately $8 \%$.

Stereo-PIV in the $(x, z)$-plane We use two PCO Sensicam with a pixel resolution of $1,280 \times 1,024$ pixel $^{2}$ in combination with the above mentioned $\mathrm{Nd}$ :YAG laser for the stereo-PIV measurements. PCO Sensicam CCD cameras have a maximum frame rate of $4 \mathrm{~Hz}$ (image pairs per second). The CCD cameras are placed upstream and downstream of the measurement area in the angular displacement method (Willert 1997; Westerweel and van Oord 2000) and the Scheimpflug condition (Prasad and Jensen 1995) is applied, see Fig. 2. The area-of-view in the $(x, z)$-plane is
$35 \times 35 \mathrm{~mm}^{2}$ in the streamwise and spanwise direction. A water prism is used to reduce the image distortion.

Raw images are again processed by means of an adaptive cross-correlation technique this time with a final interrogation area size of $32 \times 32 \mathrm{pixel}^{2}$. As in the $(x, y)$ plane, the seeding density is adjusted to three to five particles per interrogation area and the mean diameter of the seeding particles is $d_{\mathrm{m}}=50 \mu \mathrm{m}$. The accuracy of the outof-plane velocity component is somewhat lower than the accuracy of the in-plane velocity components and can be estimated to be $8 \%$.

\subsubsection{Concentration measurements}

The measurements of the concentration field are done by use of a PCO Sensicam QE with a pixel resolution of 1,376 $\times 1,040$ pixel $^{2}$. For PLIF, we use the same lens as for the PIV and stereo-PIV: Micro Nikkor $60 \mathrm{~mm}, f / 2.8$ lens (Nikon). The PCO SensicamQE allows highly spatial resolved measurements with 12bit-information but no temporal resolved measurements. The measurements of the velocity and the concentration are performed simultaneously with the same dual Nd:YAG laser. Karasso and Mungal (1997) showed the applicability of Nd:YAG lasers for quantitative LIF measurements. The areas-of-view for PLIF are approximately equal as for the PIV and stereo-PIV measurements in the respective planes.

Rhodamine B is used as fluorescent tracer dye (in aqueous solution) with an inlet concentration $c_{0}=1 \cdot 10^{-4} \mathrm{~mol} / \mathrm{l}$ and an inlet flow rate of $\dot{V}_{0}=1.5 \mathrm{ml} / \mathrm{min}$. A careful examination of the velocity field in the vicinity of the inlet shows no alteration of the flow field with and without tracer inflow, see also Fig. 9. The green 532 nm-line of the used $\mathrm{Nd}$ :YAG laser is able to sufficiently excite the tracer dye in use, see Fig. 3. Due to the fluorescent emission in the red-see Fig. 3-the emitted light from the tracer for LIF and the reflected light from the particles for PIV are easily separated by using of a highpass filter for the LIF camera
Fig. 2 Experimental setup for the combined stereo-PIV/PLIF measurements in the $(x, z)$ plane, the geometry of the channel (wave amplitude, wave length, channel height) is to scale. The PIV cameras are mounted in the angular displacement configuration where the Scheimpflug condition is applied

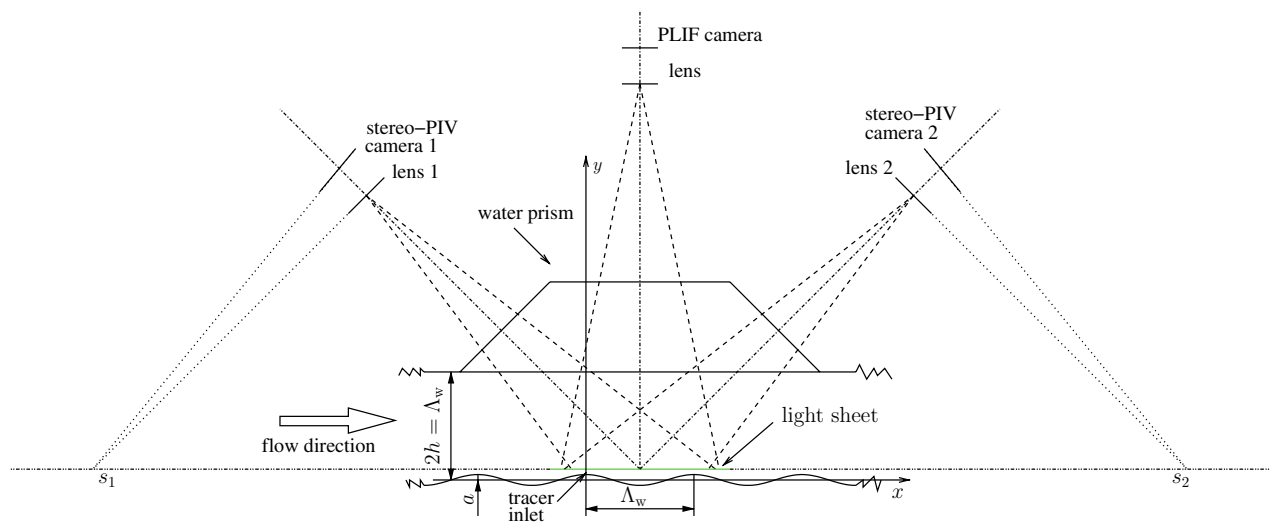




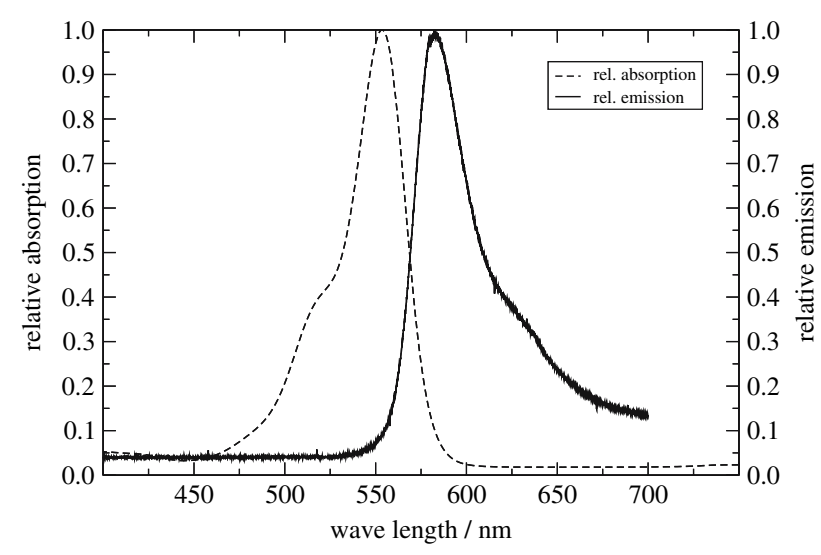

Fig. 3 Relative emission (excitation at $532 \mathrm{~nm}$ ) and relative absorption of Rhodamine $\mathrm{B}$ in aqueous solution at $20^{\circ} \mathrm{C}$

(cutoff $545 \mathrm{~nm}$ ) and a bandpass filter for the PIV camera (passing light at $532 \pm 2 \mathrm{~nm}$ ), respectively. The molecular diffusion coefficient of Rhodamine $\mathrm{B}$ in water at $20^{\circ} \mathrm{C}$ is calculated with the Wilke-Chang equation (Poling et al 2001), which results in a Schmidt number of $\mathrm{Sc} \approx 2,800$. So, even at low Reynolds numbers, the scalar transport is mostly governed by turbulent transport rather than molecular diffusion. The source is designed to have a low impact-in terms of the strength of the vertical velocity of the source and the size of the source-to the mean flow field. For ground level sources previous investigations (Fackrell and Robins 1982) showed no significant influence of the source size.

Photobleaching of the tracer dye (see Wang and Fiedler 2000) can be neglected for the present experiments. An attenuation of the incoming laser light through the tracer dye present in the water can also be neglected. During the preparation process of the experiments, the influence of the seeding density (particle density) on the PLIF measurements was investigated. It was tested how the seeding density and the reflected light from the particles affects the laser-induced fluorescence measurements. We found that the attenuation of the PLIF measurements (absorbed as well as emitted light) is not significant. The final calibration process was performed with the same seeding density than in the actual measurements. Light scattering from seeding particles does not introduce a significant amount of error to the PLIF measurements.

To establish the relationship of the local laser light intensity and the local tracer concentration

$I_{\mathrm{f}}=\phi A c I_{1}$,

at each point in the field-of-view a single pixel calibration is used. With this procedure, it is also possible to correct the inhomogeneity of the laser beam-and therefore the light sheet. In Eq. 6, $I_{\mathrm{f}}$ is the intensity of the emitted fluorescent light, $\phi$ is the quantum efficiency of the fluorescent species, the factor $A$ accounts for the optical setup and the camera characteristics, $c$ denotes the local concentration of the tracer dye, and $I_{1}$ stands for the local intensity of the laser light. Assuming a negligible time variation of the laser output energy, the unknowns in Eq. 6 are determined during the calibration process. The calibration images were acquired by taking averages of ten images for different concentrations (including zero tracer concentration to account for the dark response of the CCD cameras).

The post-processing of the raw images includes filtering and data-reduction. The data size of each image is reduced after the analysis by averaging the concentration information over an area of $4 \times 4$ pixel $^{2}$ which has been proven not to significantly alter the quality of the inherent information.

The cameras for PLIF and PIV as well as the dual cavity $\mathrm{Nd}$ :YAG laser are synchronized using a timing unit (PIVSynchronizer, ILA GmbH, Jülich) to ensure simultaneous realizations of the velocity field and the concentration field. The time-averaged data sets have been calculated from long image sequences (1,000 image pairs) obtained with a frame rate of $4 \mathrm{~Hz}$. The number of image pairs was sufficient to reach fully converged statistics.

\section{Results and discussion}

\subsection{Velocity field}

First, the results obtained with PIV at $R e_{\mathrm{b}}=5,600$ are compared with the LDV measurements of Hudson et al. (1996) $\left(R e_{\mathrm{b}}=6,760\right)$. In the work of Hudson et al the geometry parameters $\alpha$ and $\beta$ are the same as in the present study. Figure 4 shows a comparison of the mean streamwise velocity $\langle u\rangle / u_{\mathrm{b}}$ from PIV and LDV measurements. In the data sets of Hudson et al only measurements in the lower channel half are available. In order to compare the results from PIV and LDV a different definition of the bulk velocity is used which accounts only for the velocity profile in the lower channel half. The profile of the streamwise root-mean-square velocity fluctuations is given in Fig. 5. The agreement between PIV and LDV is generally good despite the fact that the measurements are obtained at somewhat different Reynolds numbers. At these lower Reynolds number values the size of the separation zone is rather sensitive to changes in the velocity. The only more significant differences in the profiles can be found in the root-mean-square velocity fluctuations $u_{\mathrm{rms}}^{\prime} / u_{\mathrm{b}}$ at the upslope of the wave profile.

In the remainder of this section, the flow field is investigated in more detail. The mean velocity field of the 


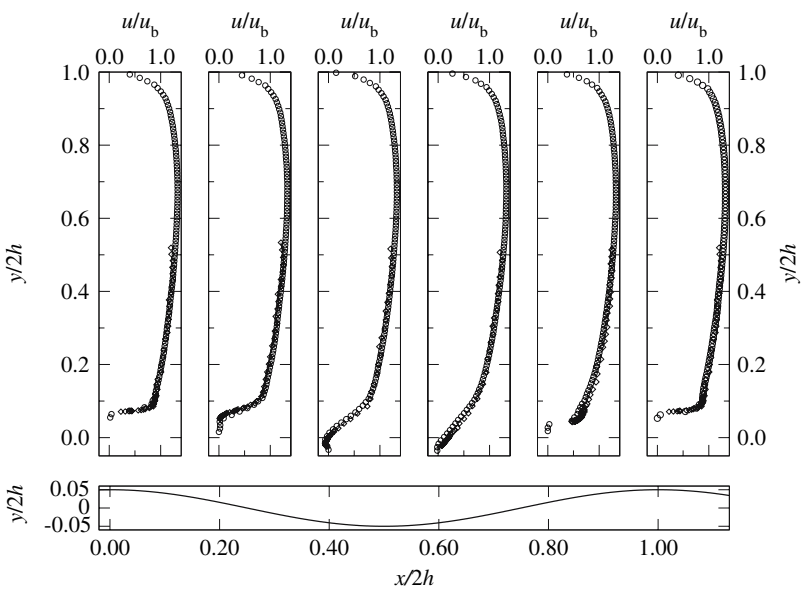

Fig. 4 Comparison of the mean streamwise velocity $\langle u\rangle / u_{\mathrm{b}}$ normalized with the bulk velocity. Results from the present PIV measurements at $R e_{\mathrm{b}}=5,600$ : circle and LDV data from Hudson et al. (1996) at $R e_{\mathrm{b}}=6,760$ : diamond. The lower graph shows the locations of the velocity profiles along the wave profile. The zero points of the velocity axes coincide with the positions at the wave

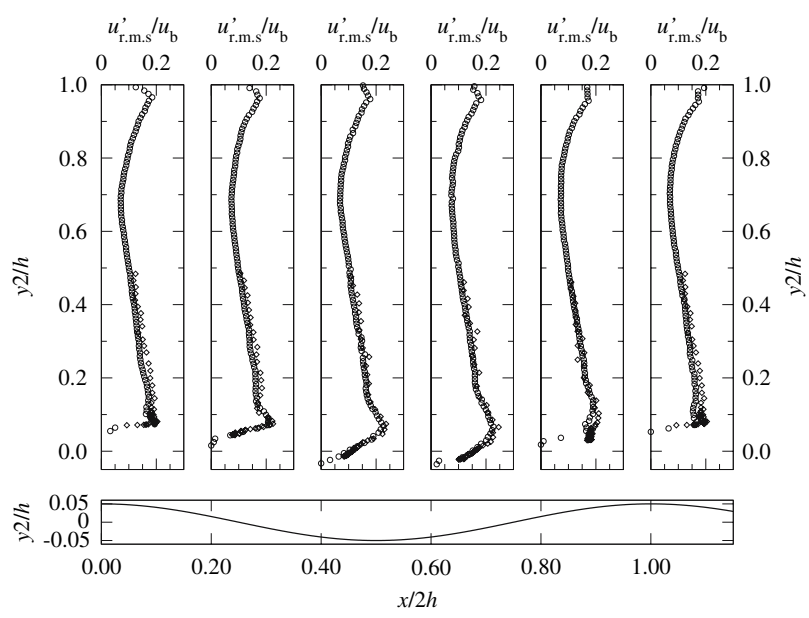

Fig. 5 Streamwise root-mean-square velocity fluctuations $u_{\mathrm{rms}}^{\prime} / u_{\mathrm{b}}$. PIV measurements at $R e_{\mathrm{b}}=5,600$ : circle and LDV data from Hudson et al. (1996) at $R e_{\mathrm{b}}=6,760$ : diamond. The lower graph shows the locations of the velocity profiles along the wave profile. The zero points of the velocity axes coincide with the positions at the wave

given flow over wavy walls (for $\alpha>0.02$ ) is mainly characterized by the recirculation zone after the wave crest and the following reattachment of the flow (Zilker et al 1977; Zilker and Hanratty 1979). Flow separation occurs shortly after the wave crest. The size of the separation zone is a function of the Reynolds number and the separation zone is less pronounced for higher values of the Reynolds number as can be seen in Fig. 6. A instantaneous realization of the velocity field is given in Fig. 9 showing the recirculation zone in the wave trough. The flow reattaches behind the wave trough in the upslope region of the wave

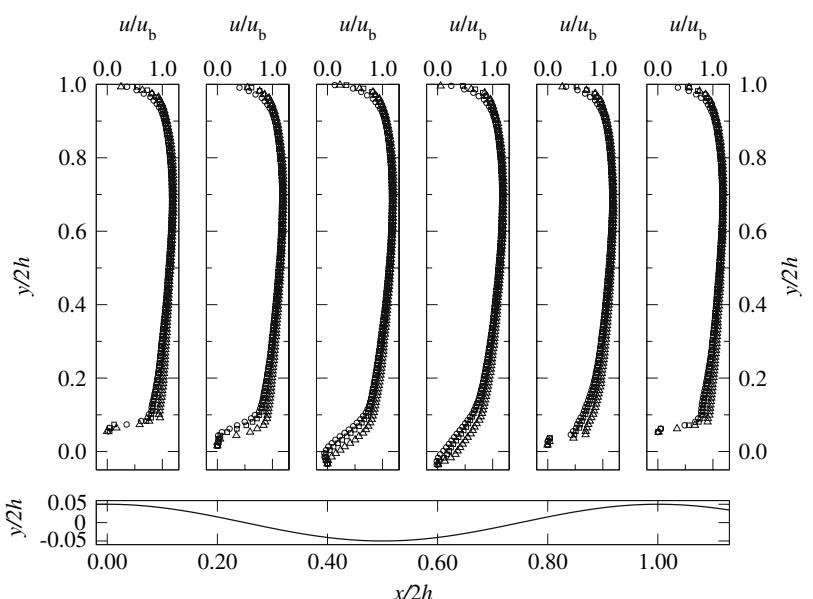

Fig. 6 Mean streamwise velocity $\langle u\rangle / u_{\mathrm{b}}$ along the wave profile. $R e_{\mathrm{b}}$ $=$ 5,600: circle, $R e_{\mathrm{b}}=11,200:$ square and $R e_{\mathrm{b}}=22,400:$ triangle. The values are normalized with the bulk velocity $u_{\mathrm{b}}$ and the channel height $2 h$. Upper $x$-axis: velocity

profile. For higher values of the Reynolds number the extent in the $y$-direction of the separation zone is reduced and the point of reattachment moves upward along the wave profile. The wavy bottom increases the intensity of the velocity fluctuations shortly behind the wave crest at the downslope region of the wave profile when compared to the flat top wall, as can be seen from Fig. 7. The high turbulence intensity increases the momentum mixing in the vicinity of the wall, accompanied with an increased pressure drop. For a more detailed description of the flow field we refer to Günther and Rudolf von Rohr (2003) and Kruse et al (2006).

\subsection{Scalar field}

\subsubsection{Instantaneous scalar distribution}

$(x, y)$-plane Two sample images of the instantaneous distribution of the scalar are given in Fig. 8. They show the scalar distribution in the $(x, y)$-plane perpendicular to the flat top wall and aligned with the streamwise direction. The concentration values are normalized with the source concentration $c_{0}$. The source is located at $x / 2 h=0.0, y / 2 h=$ 0.05 . In the $(x, y)$-plane, two 'typical' forms of the scalar structures can be distinguished. Very frequently the scalar follows in principle the geometry of the surface and the contours of the mean concentration, see Fig. 8. The dye is in this cases 'trapped' in the recirculation zone. In the same image, due to the recirculation zone, roller-like scalar structures can be clearly seen at the down-slope of the wave profile. This structures transport packets of fluid with a high scalar concentration through the domain. These regions show strong gradients of the scalar because this 


\section{(a) $u_{\mathrm{r} . \mathrm{m} . \mathrm{s} .}^{\prime} / u_{b}$}

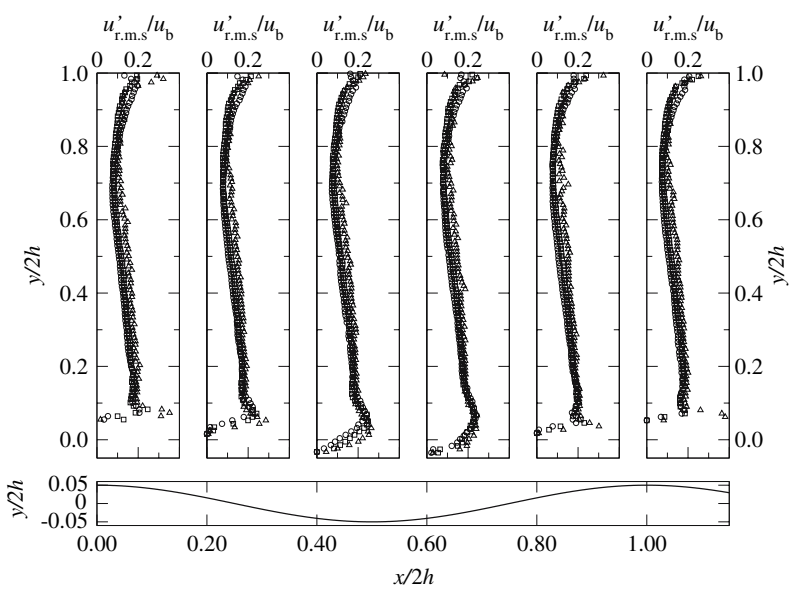

(b) $v_{\text {r.m.s. }}^{\prime} / u_{b}$

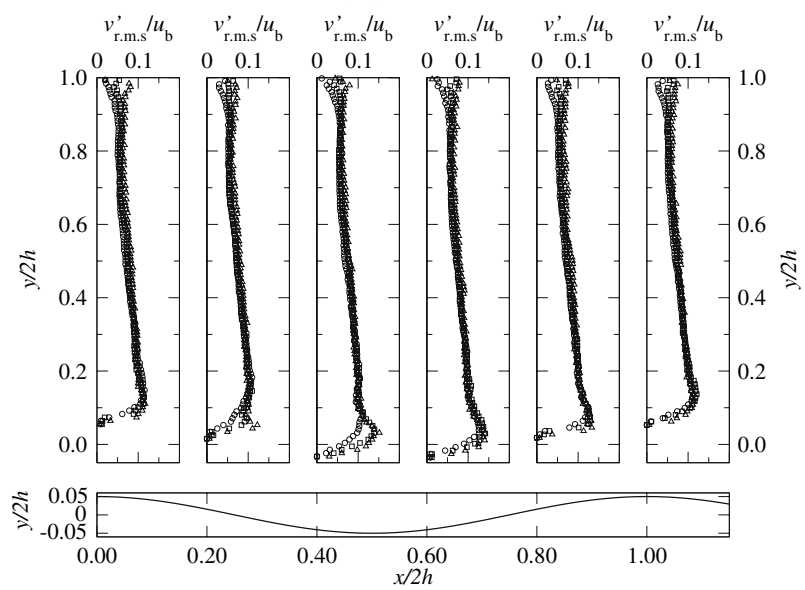

Fig. 7 Root-mean square values of the velocity fluctuations of the streamwise velocity component and the vertical velocity component. $R e_{\mathrm{b}}=5,600$ : circle, $R e_{\mathrm{b}}=11,200$ : square and $R e_{\mathrm{b}}=22,400$ : triangle. The values are normalized with the bulk velocity $u_{\mathrm{b}}$ and the channel height $2 h$. Upper $x$-axis: rms-velocities

packets place fluid with high scalar concentration in regions with nearly zero scalar concentration. The stirring and mixing processes induced by the turbulence enhance the small-scale scalar gradients which allows molecular diffusion to be more effective (Dimotakis 2005). As can be seen later in the plots of the averaged concentration, the mean scalar concentration above the wave trough is much lower than the values for the instantaneous samples. The instantaneous concentration of the tracer in the packets reaches values which are one order of magnitude higher than the mean concentration at that spatial point. In the upslope region of the wave profile the packets of fluid with a high scalar concentration disappear. The scalar distribution in this region of the flow is governed by filament-like structures. These filaments have only a limited impact on the mean scalar distribution.

The scalar transport from this kind of point source is highly unsteady. In a limited number of realization (2-3 out of 100) the scalar 'bursts' into the core of the channel flow, see Fig. 8. These scalar events are associated with similar events in the instantaneous vector fields. The velocity field shows a strong upward motion in these cases at the particular locations.

Strong intermediate upward motion above the wave crest has been reported by Günther and Rudolf von Rohr (2003). Due to the low frequency of these events they are not visible in the mean fields. A zoomed in portion of the corresponding realization of the instantaneous velocity to Fig. 8 is given in Fig. 9. The velocity field shows a strong upward motion in the region where the scalar burst is present. In comparison, the mean velocity vectors show no upward motion in this region.

$(x, z)$-plane In Fig. 10 shows two sample images of the instantaneous concentration field. The Reynolds numbers are $R e_{\mathrm{b}}=11,200$ (left) and $R e_{\mathrm{b}}=22,400$ (right), respectively. The point source is located at $x / 2 h=0.0, z / 2 h$ $=0.0$, and the concentration values are normalized with the source concentration $c_{0}$.

Snapshots of the instantaneous concentration in the $(x, z)$-plane show similar features as in the $(x, y)$-plane. Shortly after the source the scalar distribution is characterized by fluid packets with a high scalar concentration. These packets disintegrate into filaments as they proceed downstream along the wave profile. The scalar filaments appearing in Fig. 8 are also visible in Fig. 10. The visible
Fig. 8 Instantaneous concentration fields $c / c_{0}$ in the $(x, y)$-plane at a Reynolds number of $R e_{\mathrm{b}}=11,200$. The concentration values are normalized with the source concentration $c_{0}$. The scalar point source is located at $x / 2 h=$ $0.0, y / 2 h=0.05$
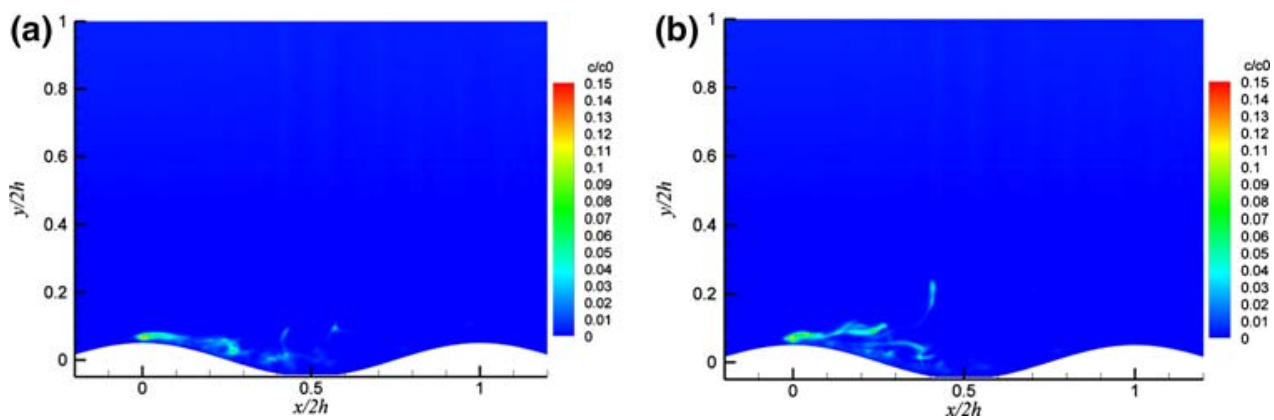


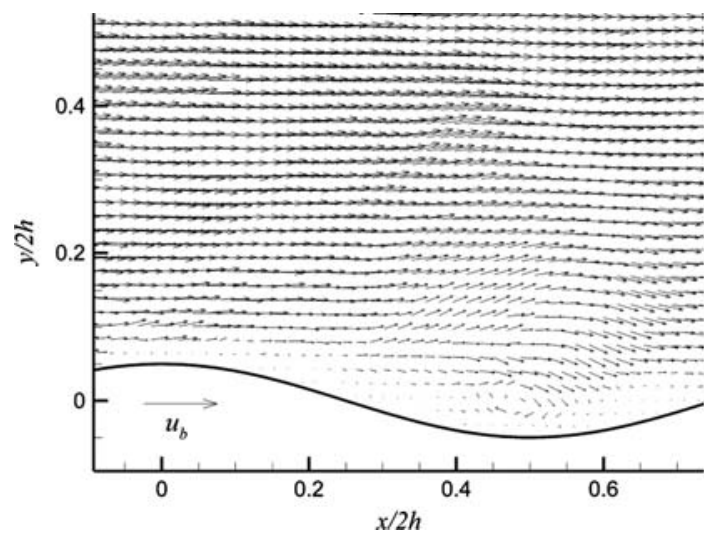

Fig. 9 Instantaneous velocity field at $R e_{\mathrm{b}}=11,200$ in the $(x, y)$-plane (zoomed in). The values of the velocity are normalized with the bulk velocity $u_{\mathrm{b}}$, reference vector below the wave profile

instantaneous structures are similar to those in scalar plumes over flat surfaces (Crimaldi et al. 2002). At higher Reynolds numbers the scalar distribution shows a narrower width than for low Reynolds numbers. Consequent realizations of the scalar field show a meandering of the tracer in the spanwise direction. The asymmetric scalar distribution in Fig. 10 results from this meandering. The turbulent transport of the scalar is mostly governed by the large-scale structures prevailing in the flow field. These large-scale structures are not stationary but meander laterally (Kruse et al. 2003). The lateral meandering of the large-scale structures is a possible explanation of the meandering of the scalar plume. From Fig. 10-regardless the fact that the snapshots are obtained at different Reynolds numbers-the unsteady nature of the scalar transport in a flow over a wavy wall is visible. From the point source, a constant volume flux of the tracer dye is released into the mean flow. The resulting scalar plume in contrast shows no continuous band of the tracer dye.

\subsubsection{Scalar statistics}

Figure 11 shows the mean concentration $\langle c\rangle$ of the scalar at a Reynolds number of $R e_{\mathrm{b}}=11,200$. The values of the

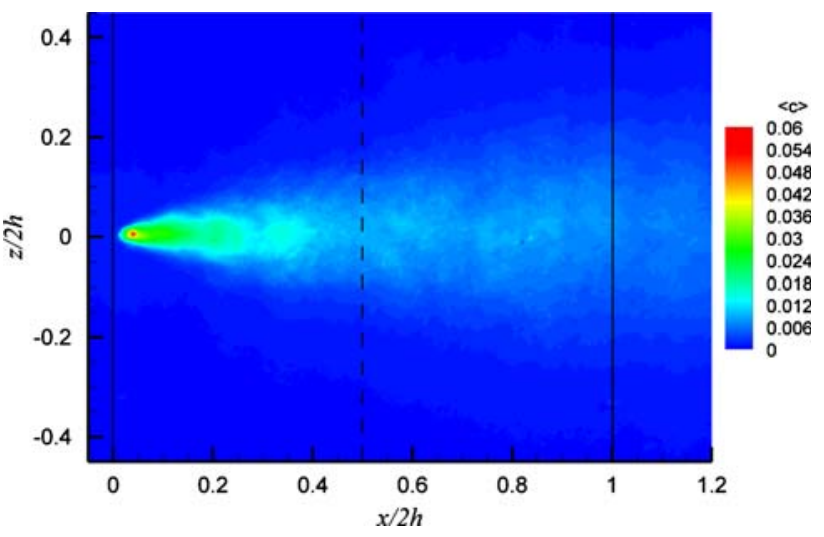

Fig. 11 Mean scalar concentration $\langle c\rangle / c_{0}$ in the $(x, z)$-plane and and $y / 2 h \approx 0.1$ at a Reynolds number of $R e_{\mathrm{b}}=11,200$, normalized with the source concentration. The source is located at $x / 2 h=0.0, z / 2 h=$ 0.0 , the solid lines indicate the wave crest and the dashed line indicates the wave trough, respectively

concentration are normalized with the source concentration $c_{0}$. The highest mean concentration is found in streamwise direction between $x / 2 h=0.05$ and $x / 2 h=0.15$. The measurement area in the $(x, z)$-plane is located at $y / 2 h=0.09$, therefore the highest values of the concentration do not appear at the source location $x / 2 h=0.0, z / 2 h=0.0$. The spreading rate of the scalar plume is somewhat higher than for a plume in a plane channel flow.

Figure 12 shows the intensity of the concentration fluctuations in the $(x, z)$-plane. The filament-like behavior of the intensity of the concentration fluctuations can be attributed to an insufficient number of realizations of the instantaneous concentration field for this kind of statistics and is not measurement noise. Due to the highly intermittent nature of the concentration field, these filaments originate mostly from single events at the specific location.

The highest intensity of the scalar fluctuations are found in the vicinity of the source which is in accordance to the findings of Fackrell and Robins (1982). In the case of a wavy wall, the intensity of the velocity fluctuations in the streamwise and wall-normal direction have their maxima at the wave crest and at the downslope of the wave profile, see Fig. 7. For the present case, there is a superimposition
Fig. 10 Instantaneous concentration fields in the $(x, z)$ plane and $y / 2 h \approx 0.1$ at Reynolds numbers of $R e_{\mathrm{b}}=$ 11,200 (left) and $R e_{\mathrm{b}}=22,400$ (right). The concentration values are normalized with the source concentration $c_{0}$. The scalar source is located at $x / 2 h=$ $0.0, z / 2 h=0.0$. The solid lines indicate the wave crest, the dashed lines the wave trough (a) $\mathrm{Re}_{\mathrm{b}}=11,200$

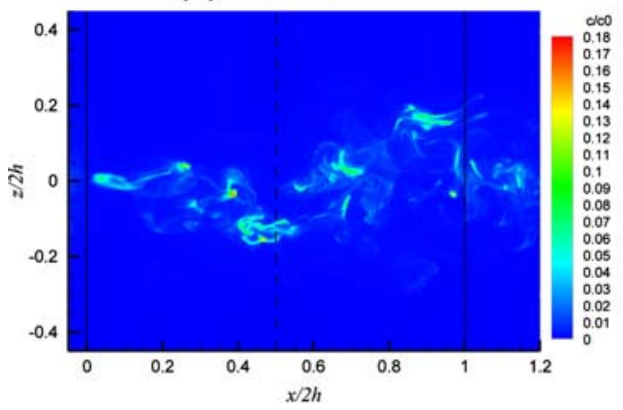

(b) $\mathrm{Re}_{\mathrm{b}}=22,400$

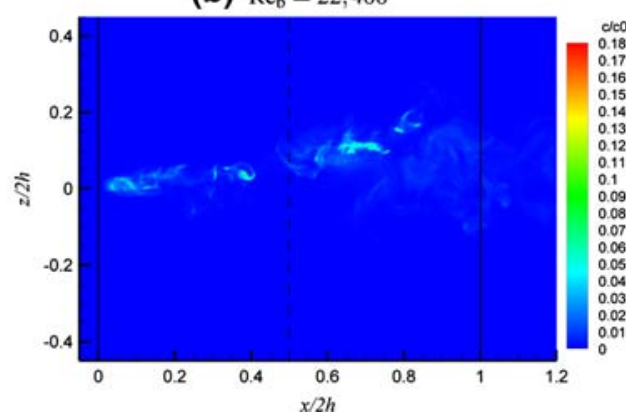




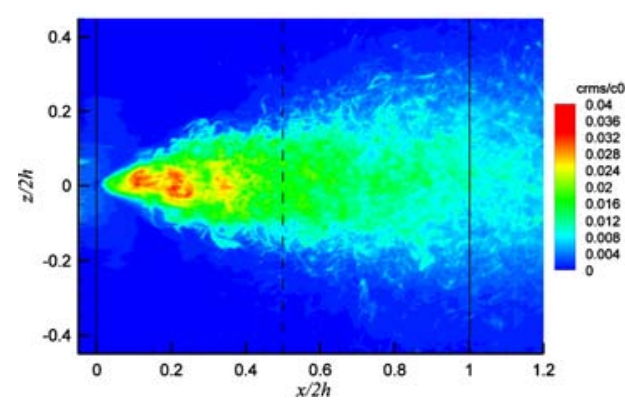

Fig. 12 Root-mean square concentration $c^{\prime}{ }_{\text {rms }} / c_{0}$ in the $(x, z)$-plane and and $y / 2 h \approx 0.1$ at $R e_{\mathrm{b}}=11,200$ (left) and $R e_{\mathrm{b}}=22,400$ (right). Values are normalized with the source concentration $c_{0}$. Source

of the high fluctuation intensities of the scalar near the source-like in a plane channel flow or flat-boundary layer flow-and the high intensity of the velocity fluctuations in the vicinity of the wave crest. This superimposition of a high intensity of the concentration fluctuations with a high turbulence intensity leads to a increased mixing rate compared to a similar point source in a plane channel flow. Figure 10 shows the mean turbulent scalar flux in the $(x, y)$-plane. The values of the turbulent scalar fluxes are normalized with the bulk velocity $u_{\mathrm{b}}$ and the source concentration $c_{0}$. In Fig. 13, the highest negative values of appear in the recirculation region. Both presented components of the turbulent scalar flux show a band of high

(a)
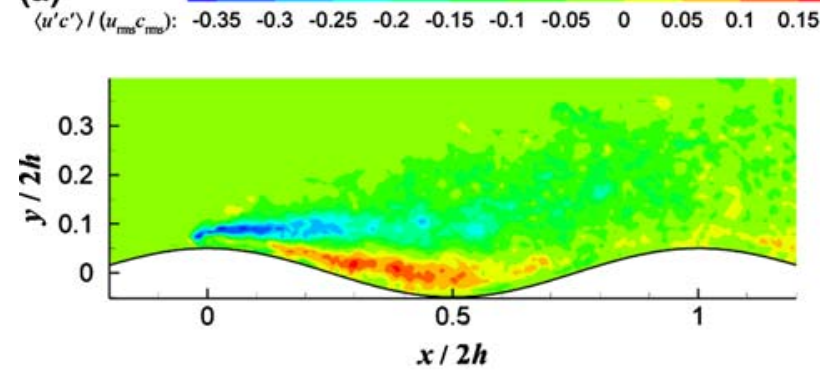

(b)
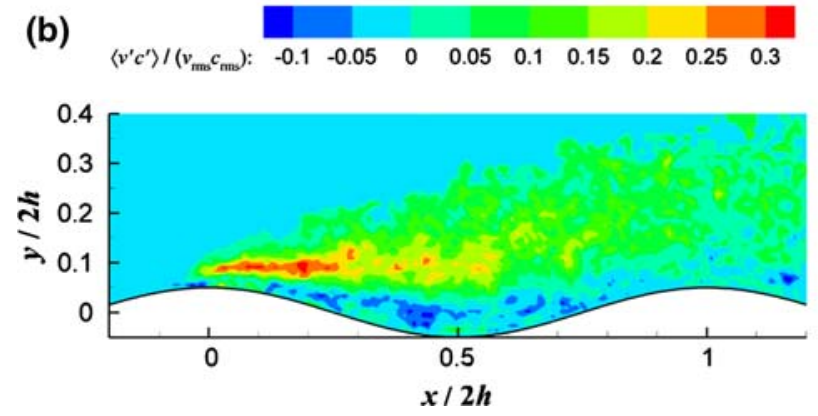

Fig. 13 Turbulent scalar flux in the $(x, y)$-plane at a Reynolds number of $R e_{\mathrm{b}}=11,200 . \mathbf{a}\left\langle u^{\prime} c^{\prime}\right\rangle /\left(u_{\mathrm{rms}} c_{\mathrm{rms}}\right), \mathbf{b}\left\langle v^{\prime} c^{\prime}\right\rangle /\left(v_{\mathrm{rms}} c_{\mathrm{rms}}\right)$. The point source is located at $x / 2 h=0.0, y / 2 h=0.05$. The values are normalized with the local root-mean-square velocity $\left(u_{\mathrm{rms}}, v_{\mathrm{rms}}\right)$ and concentration fluctuations $\left(c_{\mathrm{rms}}\right)$

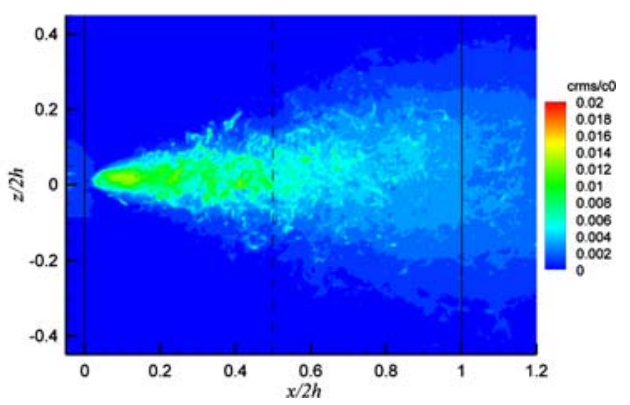

location $x / 2 h=0.0, z / 2 h=0.0$, the solid lines indicate the wave crest and the dashed lines indicate the wave trough, respectively

values of the respective scalar flux emanating from the wave crest. The highest values of the scalar fluxes are associated with high values of the turbulent momentum flux in this regions, as for example the shear layer emanating from the wave crest (see Hudson et al. 1996; Günther and Rudolf von Rohr 2003).

Figure 14 shows the streamwise and spanwise component of the scalar flux vector in the $(x, z)$-plane. The turbulent scalar flux vectors show a strong outward orientation in the spanwise direction. This leads to the an increased spreading rate of the turbulent scalar plume compared to a plume in a plane channel flow.

\subsubsection{Plume dispersion}

The evolution of the lateral plume half-width $\theta_{\mathrm{z}}$ is shown in Fig. 15. The half-width is defined as the distance in which the maximum concentration $c_{\mathrm{m}}$ falls to half its value (Fackrell and Robins 1982). For the comparison of the

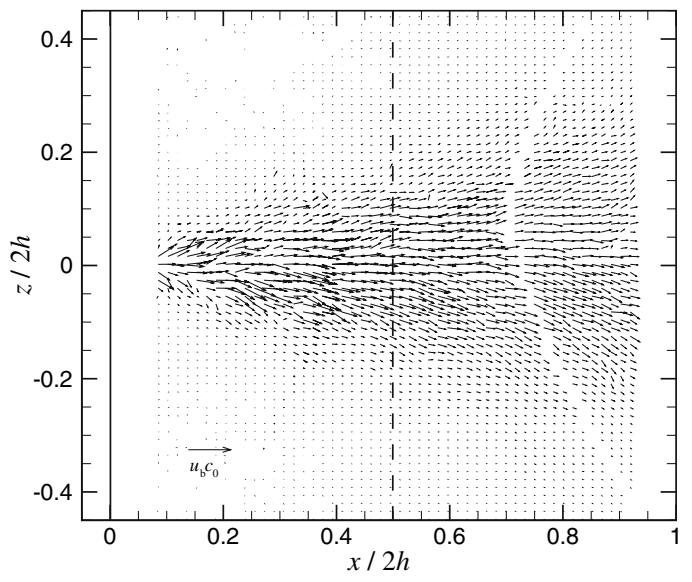

Fig. 14 Turbulent concentration flux vectors in the $(x, z)$-plane for a Reynolds number $R e_{\mathrm{b}}=11,200$ normalized with the bulk velocity and the source concentration. The scalar point source is located at $x /$ $2 h=0.0, z / 2 h=0.0$. The solid line indicates the wave crest and the dashed line indicates the wave trough, respectively. A reference vector is given in the lower left corner 


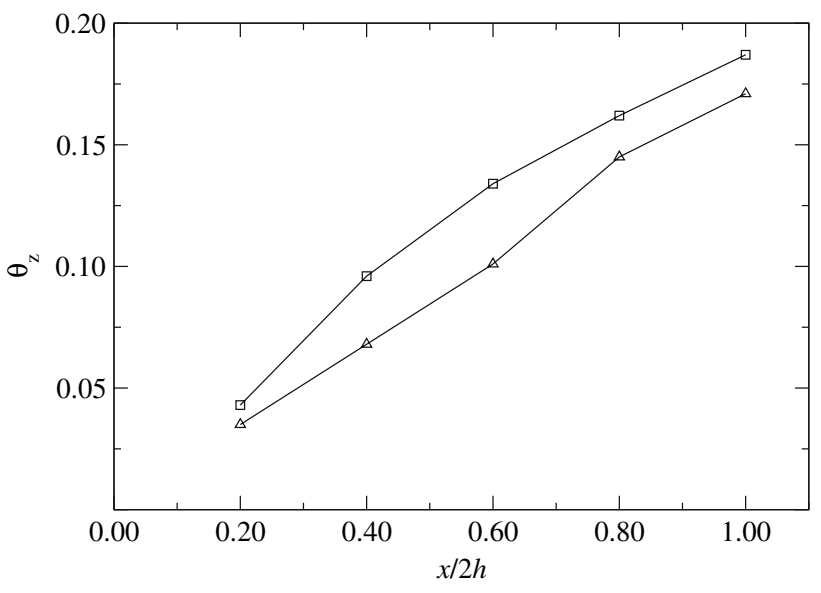

Fig. 15 Lateral plume half-width $\theta_{\mathrm{z}}$ along the wave profile. $R e_{\mathrm{b}}=$ 11,200: square and $\operatorname{Re}_{\mathrm{b}}=22,400$ triangle

results from the present study to results for plane boundary flows the vertical and lateral spreading rates of the turbulent scalar plume are defined as

$S_{\mathrm{z}}=\frac{\mathrm{d} \theta_{\mathrm{z}}(x)}{\mathrm{d} x}$.

At a Reynolds number of $R e_{\mathrm{b}}=11,200$ a lateral spreading rate of $S=0.35$ is found which is $30 \%$ higher than the spreading rate of a similar turbulent plume in a plane boundary layer (Crimaldi and Koseff 2001). The gradient of the lateral plume half width profile changes along the wave profile. In the near field of the source, the gradient $\mathrm{d} \theta_{\mathrm{z}} / \mathrm{d} x$ (or the spreading rate) is higher than in the 'far field' when the plume approaches the next wave crest. The lateral plume half width $\theta_{\mathrm{z}}$ is higher for lower Reynolds numbers and for higher Reynolds numbers, the streamwise dispersion dominates over the lateral dispersion.

Consecutive instantaneous realizations of the concentration field show a meandering of the scalar plume in the lateral direction $[(x, z)$-plane $]$ which means that the centreline position is variable in space as well as in time, see Fig. 16. No meandering in the vertical direction-as for elevated sources-can be observed in the present experiments.

In the near field of the source the lateral plume dimension is smaller than the characteristic scales of the flow structures. Kruse et al. (2006) found a lateral scale of $\mathcal{L}_{\mathrm{z}}=\mathcal{O}(3 h)$ for parameters $\alpha$ and $\beta$ as in the present study. Furthermore, the large-scale structures were found to meander laterally (Kruse et al. 2003). The lateral scale of the turbulent scalar plume is smaller than the lateral scale of the large-scale structures over the whole measurements area in the present study. It can be concluded, that the plume dynamics in the present case are mostly dominated by the dynamics of the large-scale structures prevailing in the flow field.

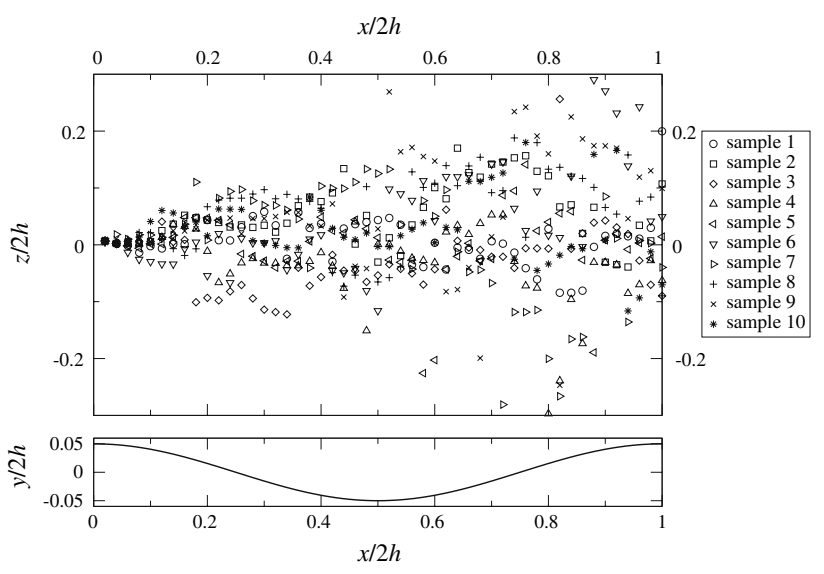

Fig. 16 Samples of the lateral plume position along the wave profile at different times (upper graph). The lower graph shows the wave profile. All co-ordinates are to scale

For a quantitative assessment of the plume dispersion, the Fickian dispersion coefficient in the lateral direction $K_{\mathrm{z}}^{\mathrm{F}}$ can be estimated from

$K_{\mathrm{z}}^{\mathrm{F}}=\frac{\left(\theta_{\mathrm{z}}^{2}(x)-\theta_{\mathrm{z} 0}^{2}\right)}{2 x / u_{\mathrm{b}}}$,

where $\theta_{\mathbf{z}}(x)$ is the lateral plume scale in a distance $x$ from the source, $\theta_{\mathrm{z} 0}$ denotes the lateral source width, and $u_{\mathrm{b}}$ is the bulk velocity which is representative for the mean advection velocity in the present case (see, e.g., Fong and Stacey 2003). Due to the changing slope of the plume-half-width profile, the Fickian dispersion coefficient will vary along the wave profile. From Eq. 8 an averaged value of $K_{\mathrm{z}}^{\mathrm{F}}=1.07 \times 10^{-4}$ $\mathrm{m}^{2} / \mathrm{s}$ is found for a Reynolds number of $R e_{\mathrm{b}}=11,200$ and for a Reynolds number of $R e_{\mathrm{b}}=22,400$ the dispersion coefficient can be estimated to $K_{\mathrm{z}}^{\mathrm{F}}=5.85 \times 10^{-3} \mathrm{~m}^{2} / \mathrm{s}$.

\section{Summary}

Measurements of the scalar transport from a low momentum turbulent plume into a turbulent flow over a wavy wall have been performed. Instantaneous realizations of the concentration field have been obtained by using PLIF whereas the velocity field is assessed by means of DPIV.

The instantaneous realizations of the scalar field show two different 'typical' scalar-distributions. The scalar transport is governed in the vicinity of the source (at the downslope of the wave profile) by roller-like structures which transport packets of fluid with high-scalar concentration into the bulk of the flow. These packets disintegrate at the upslope of the wave into filaments.

The scalar transport in the presented flow over a wavy surface is governed by the interaction of the flow field with 
the surface geometry. Two main differences between a scalar plume over a flat surface and a plume over a wavy surface can be stated. One is the lateral meandering of the scalar plume over a wavy surface which is caused by the lateral meandering of the large-scale structures apparent in the flow field. The scalar transport is therefore a highly unsteady phenomenon. The apparent large-scale structures of the velocity field allow for a very efficient mixing of the introduced tracer dye. The second difference is the influence of the separation zone. The tracer dye might be trapped in the separation zone and released as fluid packets of high scalar concentration. The high turbulence intensity in the upslope region of the wavy wall leads to an increased turbulent scalar flux in the streamwise and wall-normal direction. Therefore, the wavy wall enhances the turbulent momentum fluxes as well as the turbulent scalar flux. As a result, mixing rates are higher than in plane channel flows and the scalar plume is characterized by a higher spreading rate.

Acknowledgments Financial support from the ETH Zurich and the Swiss National Science Foundation is gratefully acknowledged. Measurement technology is partially provided by ILA GmbH, Jülich.

\section{References}

Adrian RJ (1991) Particle-imaging techniques for experimental fluidmechanics. Ann Rev Fluid Mech 23:261-304

Boyer DL, Davies PA (2000) Laboratory studies of orographic effects in rotating and stratified flows. Ann Rev Fluid Mech 32:165-202

Brederode VD, Bradshaw P (1978) Influence of side walls on turbulent center-plane boundary-layer in a square duct. Trans ASME J Fluids Eng 100:91-96

Calhoun RJ, Street RL (2001) Turbulent flow over a wavy surface: neutral case. J Geophys Res 106:9277-9293

Chang YS, Scotti A (2003) Entrainment and suspension of sediments into a turbulent flow over ripples. J Turb 4:019

Crimaldi JP, Koseff JR (2001) High-resolution measurements of the spatial and temporal scalar structure of a turbulent plume. Exp Fluids 31:90-102

Crimaldi JP, Wiley MB, Koseff JR (2002) The relationship between mean and instantaneous structure in turbulent passive scalar plumes. J Turb 3:014

Dimotakis PE (2005) Turbulent mixing. Ann Rev Fluid Mech 37:329-356

Fackrell JE, Robins AG (1982) Concentration fluctuations and fluxes in plumes from point sources in a turbulent boundary layer. J Fluid Mech 117:1-26

Fong DA, Stacey MT (2003) Horizontal dispersion of a near-bed coastal plume. J Fluid Mech 489:239-267

George WK (2007) Is there a universal log law for turbulent wallbounded flows? Phil Trans Roy Soc Lond A 365:789-806

Gong W (1991) A wind tunnel study of turbulent dispersion over twoand three-dimensional gentle hills from upwind point sources in neutral flow. Bound Lay Met 54:211-230

Gong WM, Ibbetson A (1989) A wind-tunnel study of turbulent-flow over model hills. Bound Lay Met 49:113-148
Günther A (2001) Large-scale structures in Rayleigh-Bénard convection and flow over heated waves. Dissertation, ETH Zürich

Günther A, Rudolf von Rohr P (2002) Structure of the temperature field for flow over heated waves. Exp Fluids 33:920-930

Günther A, Rudolf von Rohr P (2003) Large-scale structures in a developed flow over a wavy wall. J Fluid Mech 478:257-285

Hudson JD, Dykhno L, Hanratty TJ (1996) Turbulence production in flow over a wavy wall. Exp Fluids 20:257-265

Hussain AKMF (1983) Coherent structures-reality and myth. Phys Fluids 26:2816-2850

Karasso PS, Mungal MG (1997) PLIF measurements in aqueous flows using the Nd:YAG laser. Exp Fluids 23:382-387

Keane RD, Adrian RJ (1992) Theory of cross-correlation analysis of PIV images. Appl Sci Res 49:191-215

Krettenauer K, Schumann U (1992) Numerical simulation of turbulent convection over wavy terrain. J Fluid Mech 237:261-299

Kruse N, Rudolf von Rohr P (2006) Structure of turbulent heat flux in a flow over a heated wavy wall. Int J Heat Mass Tr 49:35143529

Kruse N, Günther A, Rudolf von Rohr P (2003) Dynamics of largescale structures in turbulent flow over a wavy wall. J Fluid Mech 485:87-96

Kruse N, Kuhn S, Rudolf von Rohr P (2006) Wavy wall effects on turbulence production and large-scale modes. J Turb 7:1-24

Maaß C, Schumann U (1996) Direct numerical simulation of separated turbulent flow over a wavy boundary. In: Hirschel EH (ed) Flow simulation with high performance computers, Notes on numerical fluid mechanics, vol 52. Vieweg, Braunschweig, pp 227-241

Poling BE, Prausnitz JM, O'Connell JP (2001) The properties of gases and liquids, 5th edn. McGraw-Hill, New York

Prasad AK, Jensen K (1995) Scheimpflug stereocamera for particle image velocimetry in liquid flows. Appl Opt 34:7092-7099

Raffel M, Willert C, Kompenhans J (2000) Particle image velocimetry, 3rd edn. Springer, Berlin

Scarano F (2002) Iterative image deformation methods in PIV. Meas Sci Tech 13:R1-R19

Sreenivasan KR (1989) The turbulent boundary layer. In: Gad-El-Hak $M$ (ed) Fontiers in experimental fluid mechanics. Springer, Berlin, pp 159-209

Walker DA (1987) A fluorescence technique for measurements of concentration in mixing liquids. J Phys E Sci Instr 20:217-224

Wang GR, Fiedler HE (2000) On high spatial resolution scalar measurements with LIF. Part 1: photobleaching and thermal blooming. Exp Fluids 29:257-264

Westerweel J (1997) Fundamentals of digital particle image velocimetry. Meas Sci Tech 8:1379-1392

Westerweel J (2000) Theoretical analysis of the measurement precision in particle image velocimetry. Exp Fluids 29:S3-S12

Westerweel J, van Oord J (2000) Stereoscopic PIV measurements in a turbulent boundary layer. In: Stanislas M, Kompenhans J, Westerweel J (eds) Particle image velocimetry. Progress toward industrial application, fluid mechanics and its applications, vol 56. Kluwer, Dordrecht, pp 11-23

Willert C (1997) Stereoscopic digital particle image velocimetry for application in wind tunnel flows. Meas Sci Tech 8:1465-1479

Zilker DP, Hanratty TJ (1979) Influence of the amplitude of a solid wavy wall on a turbulent flow. Part 2. Separated flows. J Fluid Mech 90:257-271

Zilker DP, Cook GW, Hanratty TJ (1977) Influence of the amplitude of a solid wavy wall on a turbulent flow. Part 1. Non-separated flows. J Fluid Mech 82:29-51 